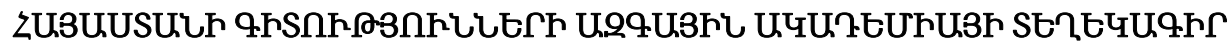 ИЗВЕСТИЯ НАЦИОНАЛЬНОЙ АКАДЕМИИ НАУК АРМЕНИИ
}

Uthuminliu

70, №2, 2017

Механика

UDC 539.3

\section{ON THE SOLUTION TO INTEGRAL EQUATIONS OF ONE CLASS OF MIXED AND CONTACT PROBLEMS BY THE DEGENERATE KERNEL METHOD}

\section{Mkhitaryan S.M.}

Key words: integral equations, degenerate kernels, contact problems, Shtaerman generalized problem, regular infinite system of equations.

Ключевые слова: интегральные уравнения, вырожденные ядра, контактные задачи, обобщённая задача Штаермана, регулярные бесконечные системы уравнений.

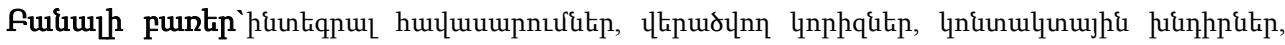

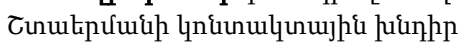

Uhuppunjuiq U.U.

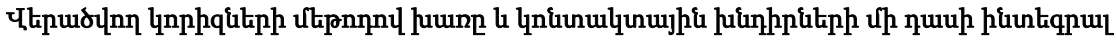

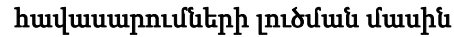

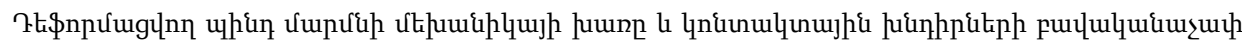

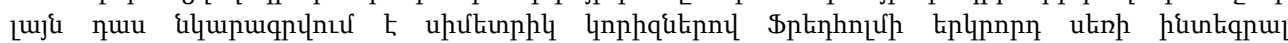

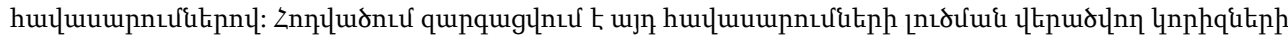

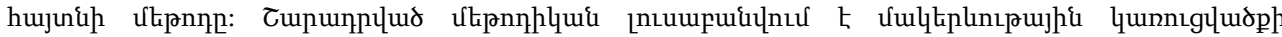

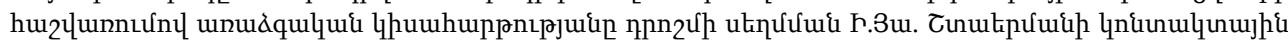

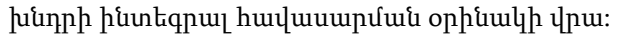

Мхитарян С.M.

О решении интегральных уравнений одного класса смешанных и контактных задач методом вырожденных ядер

Довольно широкий класс смешанных и контактных задач механики деформируемого твёрдого тела описывается интегральными уравнениями Фредгольма второго рода с симметрическими ядрами. Для решения таких уравнений в статье развивается известный метод вырожденных ядер.

Изложенная методика иллюстрируется на примере интегрального уравнения обобщённой контактной задачи И.Я. Штаермана о вдавливании штампа в упругую полуплоскость с учётом поверхностной структуры основания.

A fairly wide class of mixed and contact problems of mechanics of deformable solids is described by Fredholm integral equations of the second kind with symmetric kernels. For solving such equations, a well-known method of degenerate kernels is developed in the paper. The stated methodology is illustrated on the example of an integral equation of the E.Ja. Shtaerman generalized contact problem on indentation of a punch into an elastic halfplane taking into account the surface structure of the base.

Introduction. The method of integral equations being one of the effective methods of solution of mixed and contact problems of mechanics of deformable solids was widely applied in numerous investigations [1-9]. By the method of Green function, such problems are directly reduced to Fredholm integral equations (IE) of the first kind as well, but most 
of them can be transformed into Fredholm equations of the second kind. The latter equations can also directly arise in contact problems. This is the case in the problem of contact interaction between the elastic bodies taking into account the factor of the surface structure of the bodies contacting between each other, usually the factor of roughness by Shtaerman model of contact [1]. According to this model, because of the local deformations, the arising local displacements in each point of the contact zone are proportional to the contact stress at the very point. In such formulation in [10] an axially symmetric contact problem on indentation of a punch, circular in the plan, into a rough elastic half-space, also described by Fredholm IE of the second kind, is considered.

Numerous effective methods of solving the Fredholm IE of the second kind [11-13] are developed and among them the Fredholm method of reducing the original IE to the system of linear algebraic equations (SLAE) holds a special place. The procedure of reducing to SLAE is greatly simplified in case of degenerate kernels of IE. That is why the method of the degenerate kernels of IE solution, when the original kernel is approximated by the degenerate kernel with great exactness, has got an intensive development [11-13].

In the present paper, the method of degenerate kernels is applied to solving the Fredholm IE of the second kind with symmetrical kernels, by which integral operators with discrete spectra are generated and for these operators corresponding spectral relationship are well-known. The idea of the paper lies in the fact that based on the spectral relationship bilinear expansions of the kernels in the form of infinite series are written, then these infinite series are replaced by the finite series and, by that, the original kernels are approximated by degenerate kernels.

There is a list of symmetric kernels, for which the spectral relationship [7, 8, 14, 15] of Fredholm IE of the second kind are well known; with such kernels in the framework of the above mentioned E.Ja. Shtaerman contact model a wide class of contact problems is described. The method of degenerated kernels is concretely illustrated here on the example of E.Ja. Shtaerman generalized problem [1] on indentation of a punch of the general configuration into an elastic half-plane. It is proved that the approximate solution by the method of degenerate kernels, as the number of summands of the finite series increases infinitely, tends to the exact solution of the problem. For this purpose the issue of regularity of the corresponding infinite SLAE is investigated. In particular cases the numerical analysis of the problem is conducted.

1. General preconditions of the method of degenerate kernels.

Let us have Fredholm IE of the second kind

$$
\varphi(x)+\lambda \int_{L} K(x, s) \varphi(s) d s=f(x) \quad(x \in L)
$$

with symmetrical quadratically summarized on $L \times L$ by kernel $K(x, s)$, where $L$ is a finite or infinite interval of the numerical axis. The integral operator $K$, originated by kernel $K(x, s)(x, s \in L \times L)$, has discrete specter and for it let spectral relationships take place

$\int_{L} K(x, s) \varphi_{n}(s) w(s) d s=\lambda_{n} \varphi_{n}(x) \quad(n=0,1,2, \ldots)$.

Here $\lambda_{n}$ are eigen values, $\varphi_{n}(x)$ are eigen functions, composing full orthogonal systems in space $L_{2}(L)$, and $w(x)$ is non-negative weight function by which functions $\varphi_{n}(x)$ are orthogonal: 
$\int_{L} \varphi_{n}(x) \varphi_{m}(x) w(x) d x= \begin{cases}0 & (m \neq n) ; \\ h_{n} & (m=n) .\end{cases}$

As kernels $K(x, s)$ with above properties the following kernels can be taken

1) $\left.\ln \frac{1}{|x-s|} \quad L=(-a, a) ; 2\right) \ln \frac{x+s}{|x-s|} \quad L=(b, a) \quad(b>0)$;

3) $\ln \frac{1}{2 \sin (|x-s| / 2)} \quad L=(-\alpha, \alpha)$; 4) $\frac{1}{|x-s|^{\mu}} \quad(0<\mu<1 / 2) ; \quad L=(-a, a)$;

5) $K_{\mu}(|x-s|) /|x-s|^{\mu}(|\mu|<1 / 2) ; \quad L=(-a, a) ; \quad L=(0, \infty)$;

6) $\int_{0}^{\infty} J_{\mu}(\lambda x) J_{\mu}(\lambda s) \lambda^{2 \gamma} d \lambda(\chi \geq 0 ;|\gamma|<1 / 2) ; \quad L=(0, a)$;

7) $2 \int_{0}^{\pi} \frac{e^{-\chi_{0} \sqrt{x^{2}+s^{2}-2 x s \cos u}}}{\sqrt{x^{2}+s^{2}-2 x s \cos u}} \cos (m n) d u\left(\chi_{0}>0, m=0,1,2, \ldots\right) ; \quad L=(0, a)$.

Here $K_{\mu}(x)$ - Macdonald's function of index $\mu$ and $J_{\mu}(x)$ - Bessel's function of first kind of index $\mu$.

Fredholm IEs of the first kind with these kernels describe numerous mixed and contact problems of mechanics of deformable solids. In [7, 8, 14, 15], as well as in papers cited in $[14,15]$ for such kernels spectral relationships of type (1.2) and related to them integral relationships are established.

Now for the function $f(x)$ from $L_{2}(L)$ we write the formulas of Fourier generalized transformations in the system of functions $\varphi_{n}(x)$ :

$$
\begin{aligned}
& f(x)=\sum_{n=0}^{\infty} a_{n} \varphi_{n}(x) \quad(x \in L) \\
& a_{n}=\frac{1}{h_{n}} \int_{L} f(x) \varphi_{n}(x) w(x) d x \quad(n=0,1,2, \ldots) .
\end{aligned}
$$

Using formulas (1.4) for kernel $K(x, s)$ at fixed $x$, the following bilinear expansion of the kernel in the system of functions $\varphi_{n}(x)$ will be obtained:

$K(x, s)=\sum_{m=0}^{\infty} \frac{\lambda_{m}}{h_{m}} \varphi_{m}(x) \varphi_{m}(s) \quad((x, s) \in L \times L)$.

If in expansion (1.5) we replace the infinite sum with the finite sum restricting the number of terms by $n$, then thereby kernel $K(x, s)$ will be approximated by the degenerate kernel $K_{n}(x, s)$ :

$K(x, s) \approx K_{n}(x, s)=\sum_{m=0}^{n} \frac{\lambda_{m}}{h_{m}} \varphi_{m}(x) \varphi_{m}(s)$.

Further, in IE (1.1) kernel $K(x, s)$ is replaced by $K_{n}(x, s)$ from (1.6). After the simple transformations we shall have 


$$
\begin{aligned}
& \varphi(x)+\lambda \sum_{m=0}^{n} \frac{\lambda_{m}}{h_{m}} X_{m} \varphi_{m}(x)=f(x) \quad(x \in L) \\
& X_{m}=\int_{L} \varphi(s) \varphi_{m}(s) d s \quad(m=\overline{0, n}) .
\end{aligned}
$$

From here the approximate solution of the original IE (1.1) will be in the form of

$$
\varphi(x) \approx f(x)-\lambda \sum_{m=0}^{n} \frac{\lambda_{m}}{h_{m}} X_{m} \varphi_{m}(x) \quad(x \in L)
$$

of course, if the coefficients $X_{m}$ are already determined. For the determination of these coefficients we multiply both parts of (1.7) by $\varphi_{k}(x)(k=\overline{0, n})$ and integrate the obtained equality over the interval $L$. As a result, we come to the following SLAE:

$$
\begin{aligned}
& X_{k}+\lambda \sum_{m=0}^{n} \frac{\lambda_{m}}{h_{m}} R_{k m} X_{m}=f_{k} \quad(k=\overline{0, n}) \\
& R_{k m}=\int_{L} \varphi_{m}(x) \varphi_{k}(x) d x \quad(k, m=\overline{0, n}) ; \quad f_{k}=\int_{L} f(x) \varphi_{k}(x) d x .
\end{aligned}
$$

Thus, the method of degenerate kernels in the above described form reduces the solution of the original IE to the solution of SLAE (1.9).

Note, that in paper [10] with the help of bilinear expansion (1.5) for a symmetric kernel in the form of Veber-Sonin integral the solution of corresponding Fredholm IE of the second kind is reduced to the solution of the regular infinite SLAE. In paper [16] the method of reduction of the general class of integral equations with the symmetric quadratically summable difference or summation or difference-summation kernels to regular infinite SLAE is suggested. Moreover, by means of expanding the kernel function in Fourier cosine-series or in the series of other complete orthogonal systems of functions bilinear expansion of (1.5) type is applied. However, for the noted above class of kernels the application of expansions (1.5) in eigenvalue functions of kernels is more convenient and the use of degenerate kernels technique based on above expansions turns to be more simple.

This method is applicable to the solution of IE of I.Ja. Shtaerman generalized contact problem [1].

2. The formulation of the contact problem and derivation of basic equations. Generalizing the I.Ja. Shtaerman contact problem [1], we assume that the absolutely rigid punch, the base of which in the cross-section cut by the plane $O x y$ is described by the equation $y=f(x)$, is indented under the influence of the central vertical force $P$ and overturning moment $M$ into the elastic half-plane with Young module $E$ and Poisson coefficients $v$ (Fig.1). 


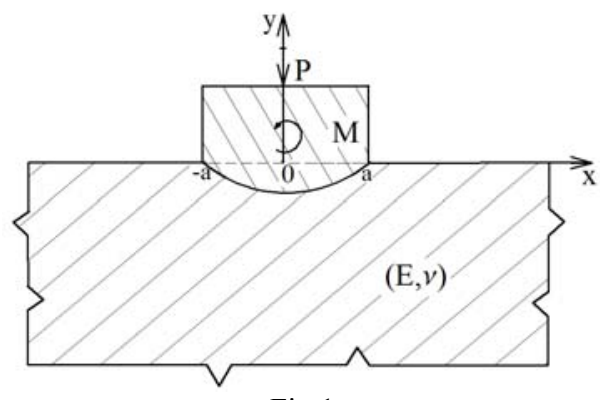

Fig.1

Here, instead of the Hertz smooth contact model we take the I.Ja. Shtaerman contact model [1] which takes into account the factor of the surface structure of deformable bodies contacting between themselves. According to this model the vertical displacements of the boundary points of the elastic half-plane are consisted of two summands. The first summand [1]

$\mathrm{v}(x)=-\vartheta \int_{-a}^{a} \ln \frac{a}{|x-s|} p(s) d s+C \quad\left(-\infty<x<\infty ; \quad \vartheta=\frac{2\left(1-v^{2}\right)}{\pi E}\right)$

arises in consequence of global deformation of the elastic body caused by the applied in the contact area $-a<x<a$ pressure $p(x)$ of the punch on the foundation in accordance with the differential equations of linear elasticity theory. The second summand $\mathrm{v}_{0}(x)$ arises in consequence of local deformations, conditioned by roughness (non-smoothness) of the contact surface, and it is considered, that at each point of the contact area it is proportional to the pressure $p(x)$ at the same point: $\mathrm{v}_{0}(x)=-\chi p(x)$, where $\chi$ is some coefficient, depending on the surface structure of the elastic body. Eventually, for the vertical displacements $\mathrm{v}_{2}(x)$ of the boundary points of the elastic half-plane we shall have

$\mathrm{v}_{2}(x)=\mathrm{v}(x)+\mathrm{v}_{0}(x)=-\chi p(x)-\vartheta \int_{-a}^{a} \ln \frac{a}{|x-s|} p(s) d s+C \quad(-a<x<a)$.

On the other hand, the vertical displacements $\mathrm{v}_{1}(x)$ of the punch, as an absolutely rigid body, have the form of

$\mathrm{v}_{1}(x)=\Delta+\alpha x \quad(-a<x<a)$,

where $\alpha$ is the angle of the rigid rotation of the punch, and $\Delta$ is its settling.

Now, substituting (2.1) and (2.2) into the contact condition [1]

$\mathrm{v}_{1}(x)-\mathrm{v}_{2}(x)=\delta-f(x) \quad(-a<x<a)$,

for the determination of the unknown contact pressure, we obtain the following Fredholm IE of the second kind:

$\chi p(x)+\vartheta \int_{-a}^{a} \ln \frac{a}{|x-s|} p(s) d s=\delta-\alpha x-f(x) \quad(-a<x<a)$.

Here, $\delta-\Delta+C$ is denoted by $\delta$.

The governing IE (GIE) (2.3) should be considered under the conditions of the punch equilibrium 


$$
\int_{-a}^{a} p(x) d x=P ; \quad \int_{-a}^{a} x p(x) d x=M .
$$

Equations (2.3)-(2.4) will be the basic equations of the considered contact problem. In them we pass to dimensionless coordinates and values, assuming

$\xi=x / a, \quad \eta=s / a ; \quad \vartheta_{0}=a \vartheta / \chi ; \quad \alpha_{0}=a \alpha / \chi E ; \quad \delta_{0}=\delta / \chi E ;$

$p_{0}(\xi)=p(a \xi) / E ; \quad f_{0}(\xi)=f(a \xi) / E \chi \quad(-1<\xi, \eta<1)$.

As a result, GIE (2.3) is transformed into the following GIE:

$p_{0}(\xi)+\vartheta_{0} \int_{-1}^{1} \ln \frac{1}{|\xi-\eta|} p_{0}(\eta) d \eta=\delta_{0}-\alpha_{0} \xi-f_{0}(\xi) \quad(-1<\xi<1)$,

and the conditions (2.4) - into the following conditions:

$\int_{-1}^{1} p_{0}(\xi) d \xi=P_{0}\left(P_{0}=P / a E\right) ; \quad \int_{-1}^{1} \xi p_{0}(\xi) d \xi=M_{0}\left(M_{0}=M / a^{2} E\right)$.

3. The solution of GIE (2.5)-(2.6) by the method of degenerate kernels. The method described in section 1 will be applied to the equations (2.5)-(2.6). In the given case $L=(-1,1)$ and the spectral relationships $(1.2)$ have the form of $[7,8,14]$

$\frac{1}{\pi} \int_{-1}^{1} \ln \frac{1}{|\xi-\eta|} \frac{T_{n}(\eta) d \eta}{\sqrt{1-\eta^{2}}}=\left\{\begin{array}{l}\frac{1}{n} T_{n}(\xi) \quad(n=1,2, \ldots) ; \\ \ln 2 \quad(n=0) ; \quad(-1<\xi<1) ;\end{array}\right.$

where $T_{n}(\xi)$ are Chebishev polynomials of the first kind, the conditions of orthogonality (1.3) has the form

$\int_{-1}^{1} T_{m}(\xi) T_{n}(\xi) \frac{d \xi}{\sqrt{1-\xi^{2}}}= \begin{cases}0 & (m \neq n) ; \\ \pi & (m=n=0) ; \quad\left(w(\xi)=1 / \sqrt{1-\xi^{2}}\right) \\ \pi / 2 & (m=n \neq 0)\end{cases}$

and the formulas of Fourier generalized transformation (1.4) have the form of

$f(\xi)=\sum_{m=0}^{\infty} f_{m} T_{m}(\xi) \quad(-1<\xi<1)$

$f_{0}=\frac{1}{\pi} \int_{-1}^{1} \frac{f(\xi) d \xi}{\sqrt{1-\xi^{2}}} ; \quad f_{m}=\frac{2}{\pi} \int_{-1}^{1} \frac{f(\xi) T_{m}(\xi) d \xi}{\sqrt{1-\xi^{2}}} \quad(m=1,2, \ldots)$.

As a result, the bilinear expansion of the kernel (1.5) in the given case for the symmetrical logarithmic kernel is written in the form of

$\ln \frac{1}{|\xi-\eta|}=\sum_{m=0}^{\infty} a_{m} T_{m}(\xi) T_{m}(\eta) \quad(-1<\xi, \eta<1) \quad a_{m}= \begin{cases}\ln 2 & (m=0) ; \\ \frac{2}{m} & (m=1,2, \ldots) .\end{cases}$

Later in accordance with (1.6), (1.8) and (1.9) in the given case the degenerate kernel is represented by the formula

$\ln \frac{1}{|\xi-\eta|} \approx K_{n}(\xi, \eta)=\sum_{m=0}^{n} a_{m} T_{m}(\xi) T_{n}(\eta) \quad(-1<\xi, \eta<1)$,

the approximate solution of GIE (2.5) is represented by the formula 
$p_{0}(\xi) \approx \delta_{0}-\alpha_{0} \xi-f_{0}(\xi)-\vartheta_{0} \sum_{m=0}^{n} a_{m} X_{m} T_{m}(\xi) \quad(-1 \leq \xi \leq 1)$,

and the unknown coefficients $X_{m}$ are determined from SLAE

$$
\begin{gathered}
X_{k}+\vartheta_{0} \sum_{m=0}^{n} a_{m} R_{k m} X_{m}=g_{k} \quad(k=\overline{0, n}) \\
R_{k m}=\int_{-1}^{1} T_{k}(\xi) T_{m}(\xi) d \xi \quad(k, m=\overline{0, n}) ; \quad g_{k}=\int_{-1}^{1} g(\xi) T_{k}(\xi) d \xi ; \\
g(\xi)=\delta_{0}-\alpha_{0} \xi-f_{0}(\xi) \quad(-1 \leq \xi \leq 1) .
\end{gathered}
$$

By the substitution $\xi=\cos t$ integrals $R_{k m}$ and $g_{k}$ are transformed into the integrals $R_{k m}=\int_{0}^{\pi} \cos k t \cos m t \sin t d t \quad(k, m=\overline{0, n})$;

$g_{k}=\delta_{0} R_{k 0}-\alpha_{0} R_{k 1}-f_{k} \quad(k=\overline{0, n}) ; \quad f_{k}=\int_{0}^{\pi} f_{0}(\cos t) \cos k t \sin t d t$

and are easily calculated. Upon that

$$
R_{k m}= \begin{cases}-\frac{1+(-1)^{m+k}}{2}\left[\frac{1}{(m+k)^{2}-1}+\frac{1}{(m-k)^{2}-1}\right](m \neq k-1 ; m \neq k+1) ; \\ 0 \quad(m=k-1 ; m=k+1) .\end{cases}
$$

From here, particularly,

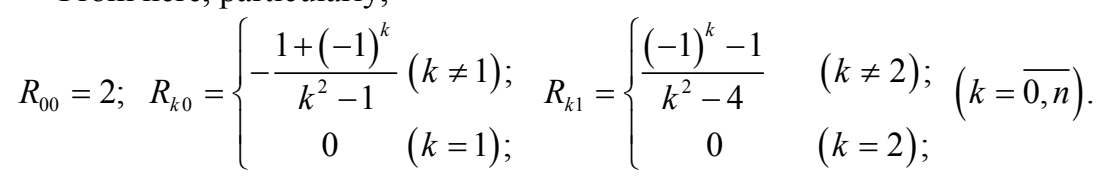

Now, taking into account the expression of the coefficients $g_{k}$ from (3.3), SLAE (3.2) is represented in the form of

$$
\begin{aligned}
& X_{k}+\vartheta_{0} \sum_{m=0}^{n} L_{k m} X_{m}=\delta_{0} R_{k 0}-\alpha_{0} R_{k 1}-f_{k} \quad(k=\overline{0, n}) \\
& L_{k m}=a_{m} R_{k m} \quad(k, m=\overline{0, n}) .
\end{aligned}
$$

Let us the solution of SLAE (3.5) for the right - hand side equal to $R_{k 0}$ denote by $X_{k}^{(1)}$, for the right - hand side $R_{k 1}$ - by $X_{k}^{(2)}$, and for the right -hand part $f_{k}$-by $X_{k}^{(3)}$.

Then solution (3.5) is represented in the form of

$$
X_{k}=\delta_{0} X_{k}^{(1)}-\alpha_{0} X_{k}^{(2)}-X_{k}^{(3)} \quad(k=\overline{0, n}) \text {. }
$$

Then, referring to the conditions of punch equilibrium (2.6), with the help of (3.1) and (3.6) for the determination of parameters $\delta_{0}$ and $\alpha_{0}$ we obtain the following SLAE:

$$
\left\{\begin{array}{l}
a_{11} \delta_{0}+a_{12} \alpha_{0}=b_{1} \\
a_{21} \delta_{0}+a_{22} \alpha_{0}=b_{2}
\end{array}\right.
$$




$$
\begin{aligned}
& a_{11}=2-\vartheta_{0} \sum_{m=0}^{n} a_{m} R_{m 0} X_{m}^{(1)} ; \quad a_{12}=\vartheta_{0} \sum_{m=0}^{n} a_{m} R_{m 0} X_{m}^{(2)} ; \\
& a_{21}=\vartheta_{0} \sum_{m=0}^{n} a_{m} R_{m 1} X_{m}^{(1)} ; \quad a_{22}=\frac{2}{3}-\vartheta_{0} \sum_{m=0}^{n} a_{m} R_{m 1} X_{m}^{(2)} ; \\
& b_{1}=P_{0}+f_{0}-\vartheta_{0} \sum_{m=0}^{n} a_{m} R_{m 0} X_{m}^{(3)} ; \quad b_{2}=-M_{0}-f_{1}+\vartheta_{0} \sum_{m=0}^{n} a_{m} R_{m 1} X_{m}^{(3)} .
\end{aligned}
$$

By equations (3.7) the dependence between the geometrical parameters of the problem $\delta_{0}$ and $\alpha_{0}$, corresponding to the reduced settlement of the punch and its reduced angle of rotation, respectively, with power parameters $P_{0}$ and $M_{0}$ is established.

Note, that taking into account (3.6) the solution (3.1) may be written in the form of

$$
\begin{aligned}
& p_{0}(\xi)=\left[1-\vartheta_{0} \sum_{m=0}^{n} a_{m} X_{m}^{(1)} T_{m}(\xi)\right] \delta_{0}-\left[\xi-\alpha_{0} \sum_{m=0}^{n} a_{m} X_{m}^{(2)} T_{m}(\xi)\right] \alpha_{0}+ \\
& +\vartheta_{0} \sum_{m=0}^{n} a_{m} X_{m}^{(3)} T_{m}(\xi) \quad(-1 \leq \xi \leq 1) .
\end{aligned}
$$

In order to investigate the convergence of the approximate solution (3.8) to the exact solution of GIE (2.5)-(2.6), it is necessary to pass from the final SLAE (3.5) to the infinite SLAE:

$$
X_{k}+\vartheta_{0} \sum_{m=0}^{\infty} L_{k m} X_{m}=\delta_{0} R_{k 0}-\alpha_{0} R_{k 1}-f_{k} \quad(k=1,2, \ldots) .
$$

Coming out from (3.4), it is easy to observe that at different parities of $k$ and $m$ we have $R_{k m}=0$. That is why in (3.4) and (3.9) $k$ and $m$ should be considered simultaneously even or odd numbers. Then the infinite system (3.9) splits up into the following two separate infinite SLAE, corresponding to the symmetric and skew-symmetric parts of the considered contact problem

$$
\begin{aligned}
& X_{2 p}+\vartheta_{0} \sum_{q=0}^{\infty} L_{2 p, 2 q} X_{2 q}=\delta_{0} R_{2 p, 0}-f_{2 p} \quad(p=0,1,2, \ldots) \\
& L_{2 p, 2 q}=a_{2 q} R_{2 p, 2 q} ; \quad a_{2 q}=\left\{\begin{array}{l}
\ln 2(q=0) \quad(k=2 p, m=2 q) ; \\
\frac{1}{q} \quad(q=1,2, \ldots) ;
\end{array}\right. \\
& X_{2 p-1}+\vartheta_{0} \sum_{q=1}^{\infty} L_{2 p-1,2 q-1} X_{2 q-1}=-\alpha_{0} R_{2 p-1,1}-f_{2 p-1} \quad(p=1,2, \ldots) \\
& (k=2 p-1, m=2 q-1) \\
& L_{2 p-1,2 q-1}=a_{2 q-1} R_{2 p-1,2 q-1} ; \quad a_{2 q-1}=\frac{2}{2 q-1} \quad(p, q=1,2, \ldots) .
\end{aligned}
$$

Here, according to (3.4) 


$$
\begin{aligned}
& R_{2 p, 2 q}=-\left[\frac{1}{4(p+q)^{2}-1}+\frac{1}{4(p-q)^{2}-1}\right] \quad(p, q=0,1,2, \ldots) ; \\
& R_{2 p-1,2 q-1}=-\left[\frac{1}{4(p+q-1)^{2}-1}+\frac{1}{4(p-q)^{2}-1}\right] \quad(p, q=1,2, \ldots) .
\end{aligned}
$$

4. The investigation of the infinite systems (3.10)-(3.11). These infinite systems will be investigated on regularity. With this aim referring to the infinite system (3.10) we estimate the sums [12]

$$
S_{2 p}=\vartheta_{0} \sum_{q=0}^{\infty} a_{2 q}\left|R_{2 p, 2 q}\right| \quad(p=0,1,2 \ldots) \text {. }
$$

Taking into account the expression $R_{2 p, 2 q}$ from (3.12), it may be written

$$
\begin{aligned}
& S_{2 p} \leq \vartheta_{0} \sum_{q=0}^{\infty} a_{2 q}\left[\frac{1}{\left|4(p+q)^{2}-1\right|}+\frac{1}{\left|4(p-q)^{2}-1\right|}\right]=\frac{2 \ln 2}{\left|4 p^{2}-1\right|} \vartheta_{0}+ \\
& +\vartheta_{0}\left[S_{2 p}^{(1)}+S_{2 p}^{(2)}\right] \quad(p=0,1,2 \ldots) ; \\
& S_{2 p}^{(1)}=\sum_{q=1}^{\infty} \frac{1}{q} \frac{1}{4(p+q)^{2}-1} ; \quad S_{2 p}^{(2)}=\sum_{q=1}^{\infty} \frac{1}{q} \frac{1}{\left|4(p-q)^{2}-1\right|} .
\end{aligned}
$$

It is evident, that

$$
\begin{aligned}
& \frac{1}{4(p+q)^{2}-1} \leq \frac{1}{4 q^{2}-1} \quad(p=0,1,2, \ldots) . \\
& \text { Therefore } \\
& S_{2 p}^{(1)}=\sum_{q=1}^{\infty} \frac{1}{q\left[4(p+q)^{2}-1\right]} \leq \sum_{q=1}^{\infty} \frac{1}{q\left(4 q^{2}-1\right)}=\sum_{q=1}^{\infty} \frac{1}{q(2 q-1)(2 q+1)}=\frac{1}{2} \ln \frac{2}{\sqrt{e}} .
\end{aligned}
$$

Here the well-known formula from [17] (p. 22, form. 0.238.1) was applied. Thus, $S_{2 p}^{(1)} \leq \frac{1}{2} \ln \frac{2}{\sqrt{e}} \quad(p=0,1,2, \ldots)$.

The sums $S_{2 p}^{(1)}$ will also be estimated with the help of Cauchy - Buniakovsky inequality:

$$
S_{2 p}^{(1)} \leq \sqrt{\sum_{q=1}^{\infty} \frac{1}{q^{2}}} \sqrt{\sum_{q=1}^{\infty} \frac{1}{\left[4(p+q)^{2}-1\right]^{2}}} \leq \frac{\pi}{\sqrt{6}} \sqrt{\sum_{q=1}^{\infty} \frac{1}{\left(4 q^{2}-1\right)^{2}}} .
$$

Calculate the sum

$$
\begin{aligned}
& \sum_{q=1}^{\infty} \frac{1}{\left(4 q^{2}-1\right)^{2}}=\frac{1}{4} \sum_{q=1}^{\infty}\left(\frac{1}{2 q-1}-\frac{1}{2 q+1}\right)^{2}= \\
& =\frac{1}{4}\left[\sum_{q=1}^{\infty} \frac{1}{(2 q-1)^{2}}-2 \sum_{q=1}^{\infty} \frac{1}{(2 q-1)(2 q+1)}+\sum_{q=1}^{\infty} \frac{1}{(2 q+1)^{2}}\right]=\frac{\pi^{2}-8}{16} .
\end{aligned}
$$


Here the expressions of these sums from [17] (p.53. form.1.444.6 and 1.444.7 for $x=0$ ) were applied. As a result,

$S_{2 p}^{(1)} \leq \frac{\pi}{4 \sqrt{6}} \sqrt{\pi^{2}-8} \quad(p=0,1,2, \ldots)$.

Based on the estimations (4.2)-(4.3), we shall have

$S_{2 p}^{(1)} \leq \min \left\{\frac{1}{2} \ln \frac{2}{\sqrt{e}}, \frac{\pi}{4 \sqrt{6}} \sqrt{\pi^{2}-8}\right\}=\frac{1}{2} \ln \frac{2}{\sqrt{e}}=0.0965736(p=0,1,2, \ldots)$.

We pass to the estimation of the sums $S_{2 p}^{(2)}$. At first note, that as above

$S_{0}^{(2)}=\sum_{q=1}^{\infty} \frac{1}{q\left(4 q^{2}-1\right)}=\frac{1}{2} \ln \frac{2}{\sqrt{e}}$.

Then, again using Cauchy-Buniakovsky inequality, we can write $(p=1,2, \ldots)$ :

$S_{2 p}^{(2)}=\sum_{q=1}^{\infty} \frac{1}{q} \frac{1}{\left|4(p-q)^{2}-1\right|} \leq \sqrt{\sum_{q=1}^{\infty} \frac{1}{q^{2}}} \sqrt{\sum_{q=1}^{\infty} \frac{1}{\left[4(p-q)^{2}-1\right]^{2}}}=\frac{\pi}{\sqrt{6}} \sqrt{\sum_{q=1}^{\infty} \frac{1}{\left[4(p-q)^{2}-1\right]^{2}}}$

Separately, estimate the sums,

$$
\begin{aligned}
& \sum_{q=1}^{\infty} \frac{1}{\left[4(p-q)^{2}-1\right]^{2}}=\sum_{q=1}^{\infty} \frac{1}{\left[4(p-q)^{2}-1\right]^{2}}+\sum_{q=p+1}^{\infty} \frac{1}{\left[4(q-p)^{2}-1\right]^{2}}= \\
& \quad(p-q=r) \quad(q-p=r) \\
& =\sum_{r=0}^{p-1} \frac{1}{\left(4 r^{2}-1\right)^{2}}+\sum_{r=1}^{\infty} \frac{1}{\left(4 r^{2}-1\right)^{2}} \leq 1+2 \sum_{r=1}^{\infty} \frac{1}{\left(4 r^{2}-1\right)^{2}} .
\end{aligned}
$$

Again using the obtained above value for the sum of the last series, we find

$S_{2 p}^{(2)} \leq \sqrt{1+\frac{\pi^{2}-8}{8}} \frac{\pi}{\sqrt{6}}=\frac{\pi^{2}}{4 \sqrt{3}} \quad(p=1,2, \ldots)$.

As

$\frac{1}{\left|4 p^{2}-1\right|} \leq 1 \quad(p=0,1,2, \ldots), \frac{1}{2} \ln \frac{2}{\sqrt{e}}<\frac{\pi^{2}}{4 \sqrt{3}}$,

then from (4.1) with the help of the estimations (4.4)-(4.6) we shall have

$S_{2 p} \leq\left(2 \ln 2+\frac{1}{2} \ln \frac{2}{\sqrt{e}}+\frac{\pi^{2}}{4 \sqrt{3}}\right) \vartheta_{0}=\left(2 \ln 2+\frac{1}{2} \ln \frac{2}{\sqrt{e}}+\frac{\pi^{2}}{4 \sqrt{3}}\right) \vartheta_{0} \quad(p=0,1,2, \ldots)$.

We require that the following condition will be fulfilled $\left(2 \ln 2+\frac{1}{2} \ln \frac{2}{\sqrt{e}}+\frac{\pi^{2}}{4 \sqrt{3}}\right) \vartheta_{0} \leq q_{0}<1$.

Whence we obtain the following condition of complete regularity [12] of the infinite system (3.10):

$$
\vartheta_{0} \leq \frac{q_{0}}{2 \ln 2+\frac{1}{2} \ln (2 / \sqrt{e})+\pi^{2} / 4 \sqrt{3}} \quad\left(0<q_{0}<1\right) .
$$


Now we shall show that for those $\vartheta_{0}$, for which the condition of complete regularity (4.7) is not fulfilled, the infinite system (3.10) is quasi-completely regular, i.e. a complete regularity in (3.10) begins with some number. For this it is sufficient to show, that $\lim _{p \rightarrow \infty} S_{2 p}=0$. Turning to the estimation of sums $S_{2 p}^{(1)}$, consider the function

$f(x)=1 / x\left[4(x+p)^{2}-1\right](x \geq 1, \quad p=0,1,2, \ldots)$.

It is evident, that the function $f(x)$ at $x \geq 1$ monotonously decreases and its value at the point $x=q(q=1,2, \ldots)$ coincides with the corresponding member of the series $S_{2 p}^{(1)}$. Herewith the sum of the series $S_{2 p}^{(1)}$, beginning from the second member, is equal to the area of the figure, consisted of the elementary rectangles with the bases of unique lengths.

Therefore,

$S_{2 p}^{(1)}<\frac{1}{4(p+1)^{2}-1}+\int_{1}^{\infty} \frac{d x}{x\left[4(x+p)^{2}-1\right]} \quad(p=0,1,2, \ldots)$.

For the calculation of this integral we use the well-known expression of the corresponding indefinite integral from [17] (p. 84, form. 2.18.4 at $m=n=1$ ). As a result,

$S_{2 p}^{(1)}<\frac{1}{4 p^{2}+8 p+3}+\frac{1}{2\left(4 p^{2}-1\right)} \times$

$\times\left[2 p \ln \left(\frac{2 p+1}{2 p+3}\right)-2 \ln 2+\ln \left(4 p^{2}+8 p+3\right)\right] \quad(p=1,2, \ldots)$.

From (4.8) it follows that $S_{2 p}^{(1)}=0\left(1 / p^{2}\right)$ at $p \rightarrow \infty$.

We pass to the estimation of the sum $S_{2 p}^{(2)}$, representing them in the form of

$S_{2 p}^{(2)}=U_{p}+\mathrm{V}_{p} ; U_{p}=\sum_{q=1}^{p} \frac{1}{q} \frac{1}{\left|4(p-q)^{2}-1\right|}=\frac{1}{p}+W_{p}$

$W_{p}=\sum_{q=1}^{p-1} \frac{1}{q} \frac{1}{4(p-q)^{2}-1} ; \quad \mathrm{V}_{p}=\sum_{q=p+1}^{\infty} \frac{1}{q} \frac{1}{4(p-q)^{2}-1} \quad(p=2,3, \ldots)$.

For the estimation of the sum $W_{p}$ we introduce to consideration the function

$h_{1}(x)=1 / x\left[4(p-x)^{2}-1\right] \quad(1 \leq x \leq p-1 ; p=3,4, \ldots)$.

It is easy to show, that this function decreases on the line segment $1 \leq x \leq p_{*}$ and increases on the line segment $p_{*} \leq x \leq p-1$, wherein

$p_{*}=\frac{1}{6}\left(4 p-\sqrt{4 p^{2}+3}\right)\left(1<p_{*}<p-1 ; p \geq 3\right)$.

Let the number $p_{*}$ be between two consecutive natural numbers $p_{0}$ and $p_{0}+1$. Then 
$W_{p} \leq \frac{1}{4 p^{2}-8 p+3}+\frac{1}{3(p-1)}+\int_{1}^{p-1} h_{1}(x) d x=\frac{1}{4 p^{2}-8 p+3}+$

$+\frac{1}{3(p-1)}+\int_{1}^{p-1} \frac{d x}{x\left[4(p-x)^{2}-1\right]} \quad(p \geq 3)$.

Passing to the estimation of the sum $\mathrm{V}_{p}$, represent them in the form of

$\mathrm{V}_{p}=\sum_{r=1}^{\infty} \frac{1}{p+r} \frac{1}{4 r^{2}-1}$

and introduce to consideration the decreasing function

$h_{2}(x)=\frac{1}{(p+x)\left(4 x^{2}-1\right)}(x \geq 1)$.

It is evident that

$\mathrm{V}_{p} \leq \frac{1}{3(p+1)}+\int_{1}^{\infty} h_{2}(x) d x=\frac{1}{3(p+1)}+\int_{1}^{\infty} \frac{d x}{(p+x)\left(4 x^{2}-1\right)}$.

Further, by the above-mentioned formula from [17] we calculate the integrals from (4.10)-(4.11). After the simple transformations we find

$U_{p} \leq \frac{1}{p}+\frac{1}{4 p^{2}-8 p+3}+\frac{1}{3(p-1)}+\frac{1}{2\left(4 p^{2}-1\right)}\{\ln [(2 p-3) \times$

$\left.\left.\times(2 p-1)(p-1)^{2}\right]-\ln 3\right\}-\frac{p}{4 p^{2}-1} \ln \frac{2 p-3}{3(2 p-1)} \quad(p \geq 4) ;$

$\mathrm{V}_{p} \leq \frac{1}{3(p+1)}+\frac{1}{4 p^{2}-1}\left[\frac{4 p+1}{2} \ln 3-\ln 2-\ln (1+p)\right]$.

Now from (4.1), (4.8), (4.9) and (4.12) it follows that $S_{2 p}=0(1 / p)$ as $p \rightarrow \infty$

and, therefore quasi-complete regularity of the infinite system (3.10) is proved. By the pretty analogous way it is possible to conduct the investigation on regularity of the infinite system (3.11).

On the base of the foregoing, the method of reduction [11] is applicable to the infinite systems (3.10)-(3.11), i.e. the solutions of the corresponding (3.10)-(3.11) of the finite SLAE as $n \rightarrow \infty$ tends to the solutions of the infinite systems.

5. Numerical results. For Poisson numerical coefficients of an elastic half-plane material we take $v=0,25$. Then the dimensionless parameter $v=0,25$ may be represented in the form of

$\vartheta_{0}=a \vartheta / \chi=\frac{15}{8 \pi} \lambda_{0} ; \lambda_{0}=a / E \chi$.

Now for the particular configurations of the punch base, when $f_{0}(\xi)=1$ or $f_{0}(\xi)=\xi^{2}$ we solve SLAE (3.5) at various values of the parameter $\lambda_{0}$. As a result, the coefficients $X_{k}^{(j)}(j=1,2,3 ; k=\overline{0, n})$ are determined and by formula (3.6) the coefficients 
$X_{k}$ are obtained. Later on from system (3.7) the parameters $\delta_{0}$ and $\alpha_{0}$ are determined, and besides it was accepted here that $P_{0}=0,001, M_{0}=0,00001$. Then using the results of these calculations, the values of the dimensionless contact pressure under the punch, $p_{0}(\xi)$, as well as the values $p_{0}( \pm 1)$ are calculated by the formula (3.8).

In case of $f_{0}(\xi)=1$ the graphs of $p_{0}(\xi)$ are practically rectilinear segments, parallel to the axis of the abscissa, which in the process of increase of $\lambda_{0}$ are removing from the axis of the abscissa. And in the case of $f_{0}(\xi)=\xi^{2}$ the graphs of $p_{0}(\xi)$ at small $\lambda_{0}$, corresponding to the big values of the local displacements in the contact zone, practically represent rectilinear segments near the axis of the abscissa. But with the increase of the parameter $\lambda_{0}$, when the local displacements become small values, the graphs of $p_{0}(\xi)$ gradually take the form of the parabola with branches going to infinity (Fig. 2).

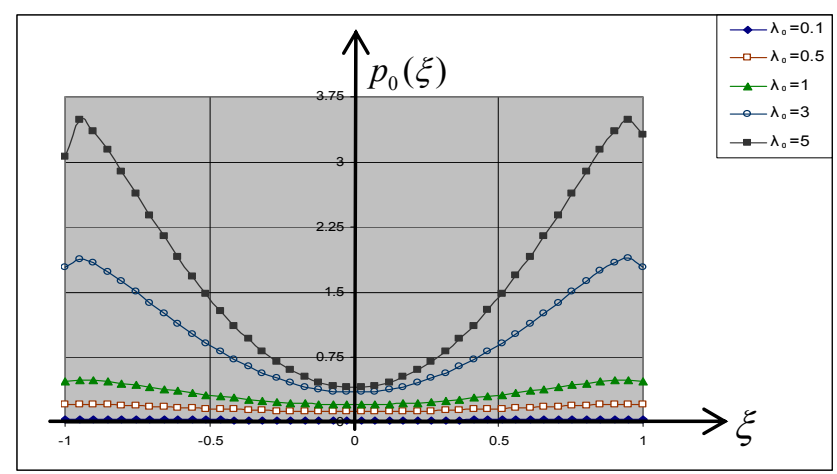

Fig. 2

Such parabolas are characteristic for the classical contact problems, when the contact pressure at the ends of the contact zone becomes infinite.

Values $\delta_{0}, \alpha_{0}$ and $p_{0}( \pm 1)$ when $f_{0}(\xi)=1$ are given in Table 1 for different $\lambda_{0}$. 
Table 1

\begin{tabular}{|r|c|c|c|r|}
\hline \multicolumn{1}{|c|}{$\lambda_{0}$} & $\delta_{0}$ & \multicolumn{1}{c|}{$\alpha_{0}$} & \multicolumn{1}{c|}{$p_{0}(-1)$} & \multicolumn{1}{c|}{$p_{0}(1)$} \\
\hline 0.001 & 0.000834 & -0.00015 & 0.000684 & 0.000984 \\
\hline 0.005 & 0.002171 & -0.00015 & 0.002022 & 0.002324 \\
\hline 0.1 & 0.034514 & -0.00016 & 0.035603 & 0.03593 \\
\hline 0.3 & 0.105856 & -0.00019 & 0.116166 & 0.116546 \\
\hline 0.5 & 0.180771 & -0.00022 & 0.207713 & 0.208146 \\
\hline 0.8 & 0.298264 & -0.00026 & 0.361064 & 0.361576 \\
\hline 1 & 0.379268 & -0.00028 & 0.471833 & 0.472396 \\
\hline 5 & 2.17879 & -0.00079 & 3.30652 & 3.30809 \\
\hline 10 & 4.57025 & -0.0014 & 7.4174 & 7.4202 \\
\hline 20 & 9.45821 & -0.00261 & 16.1845 & 16.1898 \\
\hline 50 & 24.3141 & -0.00623 & 43.8278 & 43.8403 \\
\hline 100 & 49.2167 & -0.01222 & 91.3401 & 91.3645 \\
\hline
\end{tabular}

In Table 2 the same parameters when $f_{0}(\xi)=\xi^{2}$ are represented.

Table 2

\begin{tabular}{|r|r|r|r|r|}
\hline \multicolumn{1}{|c|}{$\lambda_{0}$} & \multicolumn{1}{c|}{$\delta_{0}$} & \multicolumn{1}{c|}{$\alpha_{0}$} & \multicolumn{1}{c|}{$p_{0}(-1)$} & \multicolumn{1}{c|}{$p_{0}(1)$} \\
\hline 0.001 & 0.0015 & -0.0015 & 0.00135 & 0.00165 \\
\hline 0.005 & 0.005502 & -0.00015 & 0.005351 & 0.005652 \\
\hline 0.1 & 0.100548 & -0.00016 & 0.100366 & 0.100693 \\
\hline 0.3 & 0.300642 & -0.00019 & 0.300393 & 0.300773 \\
\hline 0.5 & 0.500736 & -0.00022 & 0.500417 & 0.50085 \\
\hline 0.8 & 0.800874 & -0.00026 & 0.800446 & 0.800958 \\
\hline 1 & 1.00096 & -0.00028 & 1.00046 & 1.00103 \\
\hline 5 & 5.00271 & -0.00079 & 5.00055 & 5.00212 \\
\hline 10 & 10.0048 & -0.0014 & 10.0004 & 10.0032 \\
\hline 20 & 20.009 & -0.00261 & 19.9999 & 20.0051 \\
\hline 50 & 50.0215 & -0.00623 & 49.9976 & 50.0101 \\
\hline 100 & 100.042 & -0.01222 & 99.9931 & 100.018 \\
\hline
\end{tabular}

With the increase of the parameter $\lambda_{0}$, which corresponds to the gradual transition into the smooth contact model, quantities $\delta_{0}$ and $p_{0}( \pm 1)$ greatly increase, while values of $\delta_{0}$ all the time remain very small.

Now we shall find out the conditions of the absence of the punch rotation when a given system of forces acts on the punch. Setting $\alpha_{0}=0$ into system (3.7), we obtain the following necessary values of $M_{0}$ and $\delta_{0}$, providing the absence of the punch rotation: 
$M_{0}=-\left\{\left[f_{1}-\vartheta_{0} Z_{n}^{(3)}\right]\left[2-\vartheta_{0} Y_{n}^{(1)}\right]+\vartheta_{0} Z_{n}^{(1)}\left(P_{0}+f_{0}-\vartheta_{0} Y_{n}^{(3)}\right)\right\} /\left(2-\vartheta_{0} Y_{n}^{(1)}\right) ;$

$\delta_{0}=\left[P_{0}+f_{0}-\vartheta_{0} Y_{n}^{(3)}\right] /\left(2-\vartheta_{0} Y_{n}^{(1)}\right)$;

$Y_{n}^{(j)}=\sum_{m=0}^{n} a_{m} R_{m_{0}} \mathrm{X}_{m}^{(j)} ; \quad Z_{n}^{(j)}=\sum_{m=0}^{n} a_{m} R_{m_{1}} \mathrm{X}_{m}^{(j)} ; \quad(j=1,2,3)$.

The calculated by the formulas (5.1) values of $\delta_{0}$ and $M_{0}$ for different $\lambda_{0}$ when $f_{0}(\xi)=1+\xi$ or $f_{0}(\xi)=\xi+\xi^{2}$ for the same value of $P_{0}$ are given in Table 3 .

\begin{tabular}{|r|r|c|c|c|}
\hline \multirow{2}{*}{$\lambda_{0}$} & \multicolumn{2}{|c|}{$f_{0}(\xi)=1+\xi$} & \multicolumn{2}{c|}{$f_{0}(\xi)=\xi+\xi^{2}$} \\
\cline { 2 - 5 } & \multicolumn{1}{c|}{$\delta_{0}$} & $M_{0}$ & $\delta_{0}$ & $M_{0}$ \\
\hline 0.001 & 0.0015 & 0.000666 & 0.000834 & 0.000666 \\
\hline 0.005 & 0.005502 & 0.003318 & 0.002171 & 0.003318 \\
\hline 0.1 & 0.100548 & 0.061202 & 0.034514 & 0.061202 \\
\hline 0.3 & 0.300642 & 0.157898 & 0.105856 & 0.157898 \\
\hline 0.5 & 0.500736 & 0.231072 & 0.180772 & 0.231072 \\
\hline 0.8 & 0.800874 & 0.312955 & 0.298264 & 0.312956 \\
\hline 1 & 1.00096 & 0.35511 & 0.379268 & 0.355111 \\
\hline 5 & 5.00271 & 0.636414 & 2.17881 & 0.636418 \\
\hline 10 & 10.0048 & 0.714155 & 4.57033 & 0.714167 \\
\hline 20 & 20.009 & 0.764888 & 9.45859 & 0.764915 \\
\hline 50 & 50.0215 & 0.802989 & 24.3169 & 0.803067 \\
\hline 100 & 100.042 & 0.818321 & 49.2273 & 0.818474 \\
\hline
\end{tabular}

From this table it is seen that at small $\lambda_{0}$, when the local displacements are significant, $\delta_{0}$ and $M_{0}$ are enough small. However, they also increase with the increase of $\lambda_{0}$.

Conclusion. Rather a wide class of contact and mixed problems of mechanics of deformable solids is described by Fredholm integral equations of the second kind with symmetric kernels, for which the corresponding integral spectral relationships are wellknown. In the paper for solving such equations, the well-known method of degenerate kernels is developed which reduces their solution to the solution of SLAE. The described method is illustrated on the example of the I.Ja. Shtaerman generalized contact problem on the punch indentation into an elastic half-plane taking into account the surface structure of the foundation. 


\section{REFERENCES}

1. Shtaerman I.Ja. Contact problem of elasticity theory. M.-L.: Gostekhteorizdat, 1949. 270p.

2. Galin L.A. Contact problems of elasticity theory and viscoelasticity. M.: 1980. 304p.

3. Vorovich E.E., Alexandrov B.M., Babeshko V.A. Nonclassical mixed problems of elasticity theory. M.: 1974. 456p.

4. Alexandrov V.M., Potarsky D.S. Nonclassical space problems of contact interactions mechanics of elastic bodies. M.: "Factorial", 1998. 288p.

5. Alexandrov V.M., Romalis B.L. Contact problems in Mechanical engineering. M.: Mechanical engineering, 1986. 174p.

6. Development of theory of contact problems in the USSR. M.: Nauka, 1976. 493p.

7. Popov G.Ja. Selected papers. V.1. Odessa: Publishing house - printing office "VMV" 2007. 438p.

8. Popov G.Ja. Selected papers. V.II. Odessa: Publishing house - printing office "VMV" 2007. 514p.

9. Hakobyan V.N. Mixed boundary - value problems on interaction of solid deformable bodies with stresses concentrators of various types. Yerevan: Publishing house "Gitutjun" NASRA, 2014. 322p.

10. Popov G.Ja., Savchuk V.V. A contact problem of elasticity theory in the presence of the contact circular area taking into account the surface structure of the contacting bodies.// Izvestia AS SSSR. MTT. 1971. №3. P.80-87.

11. Verlain A.F., Sizikov V.S. Integral equations: methods, algorithms, programs. Reference book. Kiev: Naukova dumka, 1956. 544p.

12. Kantorovich L.V., Krylov V.E. Approximate methods of higher analysis. M.-L.: Physmatgiz, 1962. 708p.

13. Trikomy F. Integral equations. M.: Publishing house EL, 1960. 299p.

14. Mkhitaryan S.M. Method of orthogonal functions in mixed problems of continuum mechanics.// In the collection of papers of the Intern. School - conf. of young scientists "Mechanics". Yerevan: 2009. P.76-93.

15. Mkhitaryan S.M. On one, connected with theory of potential, spectral correlation in spheroidal wave functions and its supplement to contact problems. // PMM. 2015. T.79. Issue 3. P.434-446.

16. Popov G.Ja. On integral equations of elasticity theory with different and summation kernels. // PMM. 1970. V.34. Issue 4. P. 603-619.

17. Gradshtein E.S., Ryjhik E.M. Tables of integrals of sums, series and compositions. M: Nauka, 1974. 1108p.

\section{Сведения об авторе:}

Mkhitaryan S.M. - Institute of Mechanics of NAS RA

E-mail: smkhitaryan39@rambler.ru

Поступила в редакцию 20.09.2016 